\title{
Nonpenetrating Deep Sclerectomy for Progressive Glaucoma: Long-term (5-year) Follow-up of Intraocular Pressure Control and Visual Field Survival
}

\author{
Grant Slagle ${ }^{1}$, Sylvia L Groth², Mario Montelongo ${ }^{3}$, William E Sponsel ${ }^{4}$
}

\begin{abstract}
Purpose: To monitor 5-year outcomes of nonpenetrating deep sclerectomy (NPDS) with mitomycin C (MMC) in a new consecutive patient cohort. Materials and methods: All eyes undergoing NPDS surgery between $1 / 08$ and $6 / 12$ were monitored for intraocular pressure (IOP), number of antiglaucoma medications (meds), and visual field indices [mean deviation (MD) and corrected pattern standard deviation (CPSD)], relative to the preoperative baseline using the two-tailed paired Student's $t$ test.

Results: Of 106 eyes undergoing NPDS with MMC, mean IOP was $19.7 \pm 0.5$ [sem] mm Hg preoperatively, $11.9 \pm 0.5$ at 3 months, $12.5 \pm 0.6$ at 6 months, $12.4 \pm 0.5$ at 12 months, $12.6 \pm 0.6$ at 18 months, $11.1 \pm 0.6$ at 2 years, $11.8 \pm 0.5$ at 2.5 years, $11.0 \pm 0.5$ at 3 years, $11.7 \pm 0.5$ at 3.5 years, $10.7 \pm 0.7$ at 4 years, $11.6 \pm 0.5$ at 4.5 years, and $12.4 \pm 0.7$ at 5 years (average IOP reduction of $7.8 \mathrm{~mm} \mathrm{Hg}$ or $37 \% ; p<10^{-6}$ ) at 5 years. About $92 \%$ of eyes had stable IOP $\geq 5$ and $\leq 21 \mathrm{~mm} \mathrm{Hg}$ at 5 years. Mean preoperative meds $2.7 \pm 0.1$ was reduced to $0.40 \pm 0.09$ at 3 months, $0.51 \pm 0.1$ at 6 months, $0.38 \pm 0.08$ at 12 months, $0.49 \pm 0.09$ at 18 months, $0.41 \pm 0.09$ at 2 years, $0.39 \pm 0.09$ at 2.5 years, $0.49 \pm 0.1$ at 3 years, $0.58 \pm 0.1$ at 3.5 years, $0.49 \pm 0.1$ at 4 years, $0.64 \pm 0.1$ at 4.5 years, and $0.52 \pm 0.1$ at 5 years, corresponding to mean reduction of 2.2 meds ( $81 \%$; $\left.p<10^{-22}\right)$ at 5 years. Mean deviation and CPSD were stable relative to baseline at all time intervals $(R=0.83-0.94 ; p<0.0001)$.

Conclusion: With appropriate postoperative management, eyes undergoing NPDS can maintain excellent IOP control with minimal medication use and maintain very stable visual fields over an extended time frame.
\end{abstract}

Keywords: Antiglaucoma, Cohort study, Glaucoma, Intraocular pressure, Medication, Mitomycin C, Nonpenetrating deep sclerectomy.

Journal of Current Glaucoma Practice (2020): 10.5005/jp-journals-10078-1273

\section{INTRODUCTION}

The ultimate objective of glaucoma surgery is to avert neurodegenerative visual field loss without compromising visual function in any other way in that process. Nonpenetrating deep sclerectomy (NPDS) is a surgical filtering procedure designed to significantly lower intraocular pressure (IOP) in eyes with openangle glaucoma while avoiding many of the collateral damage issues associated with standard trabeculectomy. ${ }^{1-4}$ Excision of the Schlemm's canal while preserving the adjacent internal layer of the trabecular meshwork (the microperforated peripheral terminus of the Descemet's membrane) reduces resistance for egress of aqueous humor without breaching the anterior chamber. Thus, IOP is lowered substantially, but hypotonous overfiltration and hyphema are avoided because the Schlemm's canal remains segregated from the anterior chamber. ${ }^{5-7}$ While avoiding postsurgical complications, NPDS also provides mean IOP reduction equivalent to that attained via trabeculectomy, and with a narrower range of variability. Nonpenetrating deep sclerectomy is technically more challenging to perform than standard trabeculectomy but can be mastered by experienced glaucoma surgeons and administered within the same surgical time frame as trabeculectomy, without added expense. . $^{8,9}$

Mitomycin C (MMC) is an antimetabolite applied intraoperatively to reduce conjunctival and scleral scarring. Mitomycin $\mathrm{C}$ has been shown to improve surgical NPDS outcomes by reducing fibrosis and hyperemia and thereby enhancing the decrease of IOP. ${ }^{1,9-12}$ This study examines the 5-year course of eyes that prior to surgery had shown rapidly progressive visual field loss on maximally tolerated
${ }^{1}$ Chicago College of Osteopathic Medicine, Midwestern University, Westmont, Illinois, USA

${ }^{2}$ Department of Ophthalmology Glaucoma Service, Vanderbilt University, Nashville, Tennessee, USA

${ }^{3}$ Glaucoma Service, WESMDPA Baptist Medical Center, San Antonio, Texas, USA

${ }^{4}$ Department of Vision Sciences, University of the Incarnate Word, San Antonio, Texas, USA

Corresponding Author: William E Sponsel, Department of Vision Sciences, University of the Incarnate Word, San Antonio, Texas, USA, Phone: +1 (210) 275-8663, e-mail: sponsel@earthlink.net

How to cite this article: Slagle G, Groth SL, Montelongo M, et al. Nonpenetrating Deep Sclerectomy for Progressive Glaucoma: Longterm (5-year) Follow-up of Intraocular Pressure Control and Visual Field Survival. J Curr Glaucoma Pract 2020;14(1):3-9.

Source of support: Nil

Conflict of interest: None

medical therapy, all of which underwent MMC-augmented NPDS with serial monitoring of IOP, antiglaucoma medication (meds) use, and the Humphrey visual field (HVF) status.

\section{Materials and Methods}

This Institutional Review Board (IRB)-approved retrospective quality assurance study includes all eyes of patients undergoing planned NPDS surgery between January 2008 and June 2012 at a private

() The Author(s). 2020 Open Access This article is distributed under the terms of the Creative Commons Attribution 4.0 International License (https://creativecommons. org/licenses/by-nc/4.0/), which permits unrestricted use, distribution, and non-commercial reproduction in any medium, provided you give appropriate credit to the original author(s) and the source, provide a link to the Creative Commons license, and indicate if changes were made. The Creative Commons Public Domain Dedication waiver (http://creativecommons.org/publicdomain/zero/1.0/) applies to the data made available in this article, unless otherwise stated. 
practice in South Texas (a different cohort than was reported upon earlier in our 2013 medium-term follow-up study). ${ }^{11}$ All eyes undergoing NPDS were provisionally consented to undergo either NPDS or trabeculectomy. Types of glaucoma included primary open and closed angle, congenital, and neovascular. During surgery, eyes that were observed to have spontaneous peaking of the pupil or visible peripheral iris prolapse were converted to full-thickness trabeculectomy with peripheral iridectomy. All such eyes were excluded from this analysis.

After successful NPDS, even among eyes with an intact internal trabecular meshwork (TM), iris root incarceration can inhibit filtration of aqueous humor and lead to increased IOP. This problem can be reversed via intracameral injection of acetyl choline (Miostat, Alcon, Fort Worth, TX) as an outpatient slit-lamp procedure. The rapidly induced miosis pulls the iris away from the iridocorneal junction, thereby restoring filtration. When this maneuver proved necessary, it was followed by a 2-month course of topical $2 \%$ pilocarpine to prevent recurrence. All instances of this or any other clinic-based filtration-preserving maneuver were documented and tallied.

All eyes were regularly assessed for IOP by a calibrated slit lamp using slit lamp-mounted Goldmann applanation tonometry. Topical medication use and scotopic visual acuity (Acuity System 3.8 , Temeculah, CA) were also performed prior to surgery, at 3 months ( \pm 2 weeks) postoperatively, and every $6( \pm 2)$ months postoperatively for 5 years. Humphrey Visual Field Analyzer II 30-2 SITA full threshold (HVF; Humphrey Zeiss, Dublin, CA) perimetric testing was performed and mean deviation (MD) and pattern standard deviation (PSD) documented. These tests were typically undertaken less frequently than tonometry and medication monitoring. For this reason, our data were analyzed in two contemporaneous time-coordinate sets, one for IOP and medications and the other for HVF 30-2 MD and corrected pattern standard deviation (CPSD).

Antiglaucoma medications, both topical and oral, were documented at each visit. Antiglaucomatous agents administered in topical combination formulations were counted as two medications. Topical carbonic anhydrase inhibitors administered as monotherapy in eyes with well-controlled post-NPDS IOP values below $15 \mathrm{~mm} \mathrm{Hg}$ were not considered to be ocular antihypertensive medications when their documentation indicated the drug was added to enhance retinal circulation via its IOP-independent vascular effects. ${ }^{11,13}$ Visual acuity was measured under scotopic conditions using standard Snellen letters on a wall-mounted monitor screen. Visual fields were tested using Humphrey II visual field (HVF) 30-2 full threshold static perimetry. Only data from subjects yielding HVFs with fixation loss, false-positive, and falsenegative rates $<25 \%$ and had visual acuity better than $20 / 80$ at each visit were included for analysis of MD and CPSD.

Surgeries were performed on the more diseased eye of patients providing a signed informed consent after discussion of the potential risks and benefits of undergoing MMC-augmented NPDS. The chief steps of the procedure were as follows: after placement of $7 \mathrm{~mL}$ of 50/50 lidocaine/bupivacaine seventh nerve and retrobulbar 50/50 lidocaine/bupivacaine anesthetic blocks around the operative eye, a silk superior rectus stay suture was placed to rotate the globe into downgaze. A posterior conjunctival Westcott scissor incision was then formed immediately anterior to the stay suture over the superior rectus muscle, relaxed laterally and medically anterior to the fornix, followed by an offset tenons capsule and episcleral dissection anterior the superior rectus muscle insertion. Blunt dissection was performed to debride the episclera, and once the intact posterior limbus was revealed, 23-gauge electrocautery was used to attain hemostasis. A $4 \times$ $6 \mathrm{~mm}$ flap at $1 / 3$ scleral depth was then created using a ruby crescent blade (Huco Vision SA; St Blaise, Switzerland). An internal scleral flap was then demarcated to $90 \%$ scleral depth with a diamond blade, and then dissected in the laminar fashion just above the ciliary body with the ruby blade. The inner flap was then separated from the trabecular meshwork and the associated peripheral Descemet's membrane using a diamond blade. The Schlemm's canal was then unroofed using Mermoud microforceps (Huco Vision SA; St. Blaise, Switzerland), typically resulting in spontaneous egress of aqueous humor without significant change in anterior chamber depth or iris planarity. Three $0.3 \mathrm{~mL}$ doses of antimetabolite were topically applied using $0.4 \mathrm{mg} / \mathrm{mL}$ MMC saturated cellulose sponges for 1 minute each: one within the scleral lake, one laterally, and another medially over the flap beneath the limbal zone of the tenoconjunctival flap. The operative site was then irrigated with $3 \mathrm{~mL}$ of saline and the external scleral flap was sutured closed using 10-0 nylon suture at its posterior corners. The Tenons capsule was closed using continuous locking 8-0 polygalactin on a tapered BV needle [Vicryl; Ethicon (Johnson \& Johnson), New Brunswick, NJ], and the conjunctiva was closed in the opposite direction using tightly spaced continuous nonlocking bites with the same suture in the reverse direction, resulting in a spontaneously elevating watertight bleb. Photographic depiction of the key steps of the procedure can be seen in our previous publication. ${ }^{11}$

The eye was then treated with polymyxin/neomycin/ dexamethasone ointment, and a patch and Fox shield were positioned over the eye. All patients were examined the following morning at the slit lamp. A 1-month postoperative taper of topical prednisolone acetate 1.0\% (Omnipred; Alcon, Fort Worth, TX) and moxifloxacin 0.5\% (Vigamox; Alcon, Fort Worth, TX) was prescribed, four drops per day week 1, three per day week 2, etc. Follow-up visits were routinely scheduled 1 day, 1 week, 3 weeks, 3 months, and every 6 months thereafter.

Any further clinical or surgical manipulations done to enhance or preserve the NPDS bleb during the 5-year follow-up period were documented. Each tracked variable (IOP, number of antiglaucoma medications, MD, and (PSD) was statistically evaluated and compared to the relevant preoperative baseline value using the two-tailed paired Student's $t$ test. In all instances, comparisons were made only comparing baseline and follow-up values between the same precise cohort of eyes being followed up at each time interval.

The Bonferroni Correction was applied to ensure that all determinations of statistical significance are indeed significant and not due to chance related to the number of unplanned hypotheses tested. Because comparisons were made at each of 11 time intervals for IOP, number of medications, MD, and CPSD, 44 Students' $t$ tests were calculated. Therefore, to ensure $\alpha=0.05$, the $p$ values among this study group must be less than 0.001 in order to be considered statistically significant. ${ }^{14}$

Complete and qualified success rates were also examined. As commonly described in other studies, ${ }^{15-17}$ complete success was defined as IOP greater than $4 \mathrm{~mm} \mathrm{Hg}$ and less than $21 \mathrm{~mm} \mathrm{Hg}$ with no antiglaucoma medications at 5 years; qualified success was defined as IOP greater than $4 \mathrm{~mm} \mathrm{Hg}$ and less than $21 \mathrm{~mm} \mathrm{Hg}$ with medical glaucoma treatment. Failure was defined as IOP less than $5 \mathrm{~mm} \mathrm{Hg}$ or greater than $21 \mathrm{~mm} \mathrm{Hg}$ at 5 years. Eyes requiring ancillary procedures such as miostat injections were included for 
consideration as a complete or qualified success. Note that eyes with stable IOP less than $15 \mathrm{~mm} \mathrm{Hg}$ on carbonic anhydrase inhibitor monotherapy for IOP-independent circulatory effects as noted in the medical record were considered to be on zero IOP-lowering medications.

Reasons for attrition and potential resultant selection bias were investigated. ${ }^{18}$ Data recorded at the latest data point of attritional patients were compared with nonattritional eyes at the same interval.

\section{Results}

During the defined study period, 166 eyes of 123 patients underwent filtering surgery with the current procedural terminology (CPT) code 66172 (glaucoma filtering procedure with intraoperative antimetabolite administration). Types of glaucoma were primary open angle, narrow angle, intermittent angle closure, juvenile, congenital, neovascular, and uveitic. Of these, 44 eyes were either intentionally performed as planned trabeculectomy or converted to full-thickness trabeculectomy for reasons indicated earlier. For comparison of baseline characteristics of eyes converted to trabeculectomy, see Table 1. Of those undergoing NPDS or trabeculectomy, 13 were modified during the 5-year follow-up with tube shunts, and 3 NPDS eyes underwent late surgical iridectomy because of iris incarceration. These were excluded from the final analysis, leaving 106 eyes qualifying for the study inclusion criteria. Among these, 42 eyes required ancillary procedures, including 22 clear cornea phacoemulsification cataract extractions, with or without associated YAG laser capsulotomies, and 13 injections of

Table 1: Baseline comparison of eyes receiving nonpenetrating deep sclerectomy and those converted to trabeculectomy

\begin{tabular}{|c|c|c|c|c|}
\hline & Age (years) & Male:female & $\begin{array}{l}\text { Preoperative } \\
\text { intraocular } \\
\text { pressure }\end{array}$ & $\begin{array}{l}\text { Preoperative } \\
\text { medications }\end{array}$ \\
\hline NPDS eyes & 61.8 & $16: 41$ & 19.8 & 2.76 \\
\hline $\begin{array}{l}\text { Trabeculec- } \\
\text { tomy eyes }\end{array}$ & 70 & 06:07 & 32.9 & 3.07 \\
\hline$p$ & $<0.01$ & & $<0.01$ & 0.32 \\
\hline
\end{tabular}

Miostat in 9 eyes to resolve transient iris incarceration as described earlier. Eyes without preoperative baseline pressure/medication or comprehensive sets of highly reliable visual field data were also excluded from the respective final analyzes for these variables. This left a total of 96 eyes for the medication/IOP portion of the analysis, and 42 eyes for the visual field (MD/CPSD) portion.

Reduction of IOP and antiglaucoma medication was noted at every postoperative interval up to and including 5 years (Fig. 1). Mean IOP with standard error of the mean was $19.7 \pm 0.5 \mathrm{~mm} \mathrm{Hg}$ preoperatively, $11.9 \pm 0.5$ at 3 months postoperatively, $12.5 \pm 0.6$ at 6 months, $12.4 \pm 0.5$ at 12 months, $12.6 \pm 0.6$ at 18 months, $11.1 \pm$ 0.6 at 2 years, $11.8 \pm 0.5$ at 2.5 years, $11.0 \pm 0.5$ at 3 years, $11.7 \pm 0.5$ at 3.5 years, $10.7 \pm 0.7$ at 4 years, $11.6 \pm 0.5$ at 4.5 years, and $12.4 \pm$ 0.7 at 5 years. For the final data point at 5 years' postoperation, this corresponds to an average IOP reduction of $7.8 \mathrm{~mm} \mathrm{Hg}$ or $37 \%$ $\left(p<10^{-6}\right)$. Altogether $92 \%$ had stable IOP $\geq 5$ and $\leq 21 \mathrm{~mm} \mathrm{Hg}$ at 5 years.

The mean number of preoperative antiglaucoma medications was $2.7 \pm 0.1$, reduced to $0.40 \pm 0.093$ months postoperatively, 0.51 \pm 0.1 at 6 months, $0.38 \pm 0.08$ at 12 months, $0.49 \pm 0.09$ at 18 months, $0.41 \pm 0.09$ at 2 years, $0.39 \pm 0.09$ at 2.5 years, $0.49 \pm 0.1$ at 3 years, $0.58 \pm 0.1$ at 3.5 years, $0.49 \pm 0.1$ at 4 years, $0.64 \pm 0.1$ at 4.5 years, and $0.52 \pm 0.1$ at 5 years (Fig. 2). At 5 years, this corresponded to an average reduction of 2.2 medications (81\%), with a $p$ value $<10^{-22}$.

The complete success rate related to IOP and antiglaucoma medication use (no medication and IOP $>4<21 \mathrm{~mm} \mathrm{Hg}$ ) was determined to be $70 \%$ at 5 years, and the qualified success rate (the same with any level of topical medication) was $87 \%$ (Fig. 3). Of the nine eyes that received miostat injections, six had data at 5 years. Five of these eyes qualified as complete success, and one met criteria as a qualified success. These patients were significantly older than those not requiring miostat injections, but no difference was observed in pre- or postoperative IOP, medications, or visual field parameters (Table 2 ).

The failure rate was 4/52 (7.7\%). Two eyes had hypotony (IOP $<4)$ and two had IOP $>21$. All four had pressure-medication data at 5 years and two had visual field data. There was no significant difference in age at operation, preoperative IOP, or preoperative medications when compared to eyes that did have adequately controlled 5-year IOP ( $p=0.75,0.85$, and 0.99 , respectively).

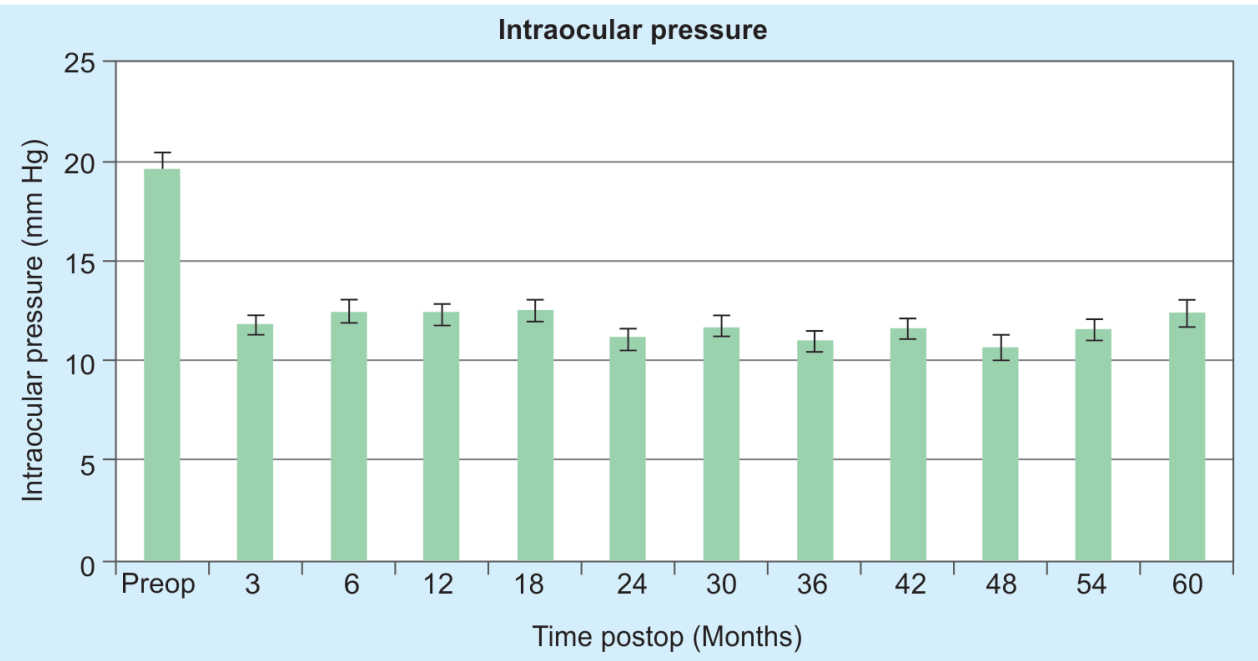

Fig. 1: Average intraocular pressure (IOP) at each time interval of patients with nonpenetrating deep sclerectomy modified with mitomycin C. Error bars show standard error of the mean, the $p$ value for significant difference between preoperative IOP and IOP at each time interval are all $<10^{-6}$ 


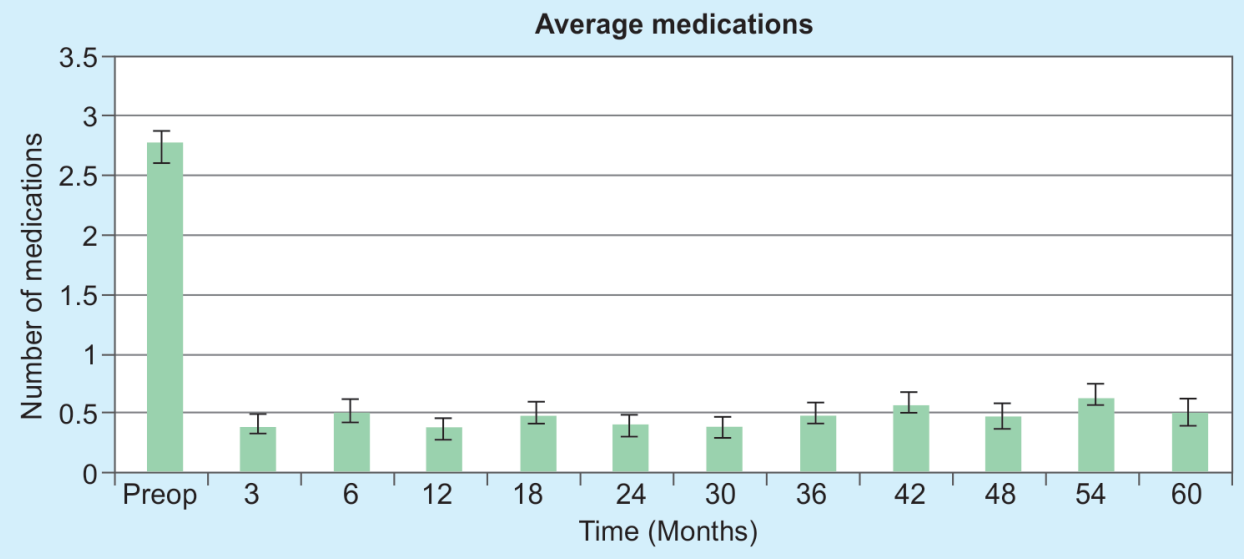

Fig. 2: Mean reduction in number of antiglaucoma medications used in patients with nonpenetrating deep sclerectomy modified with mitomycin C. Error bars represent standard error of the mean, $p$ values for significant difference between preoperative medications and each postoperative time point are all $<10^{-14}$

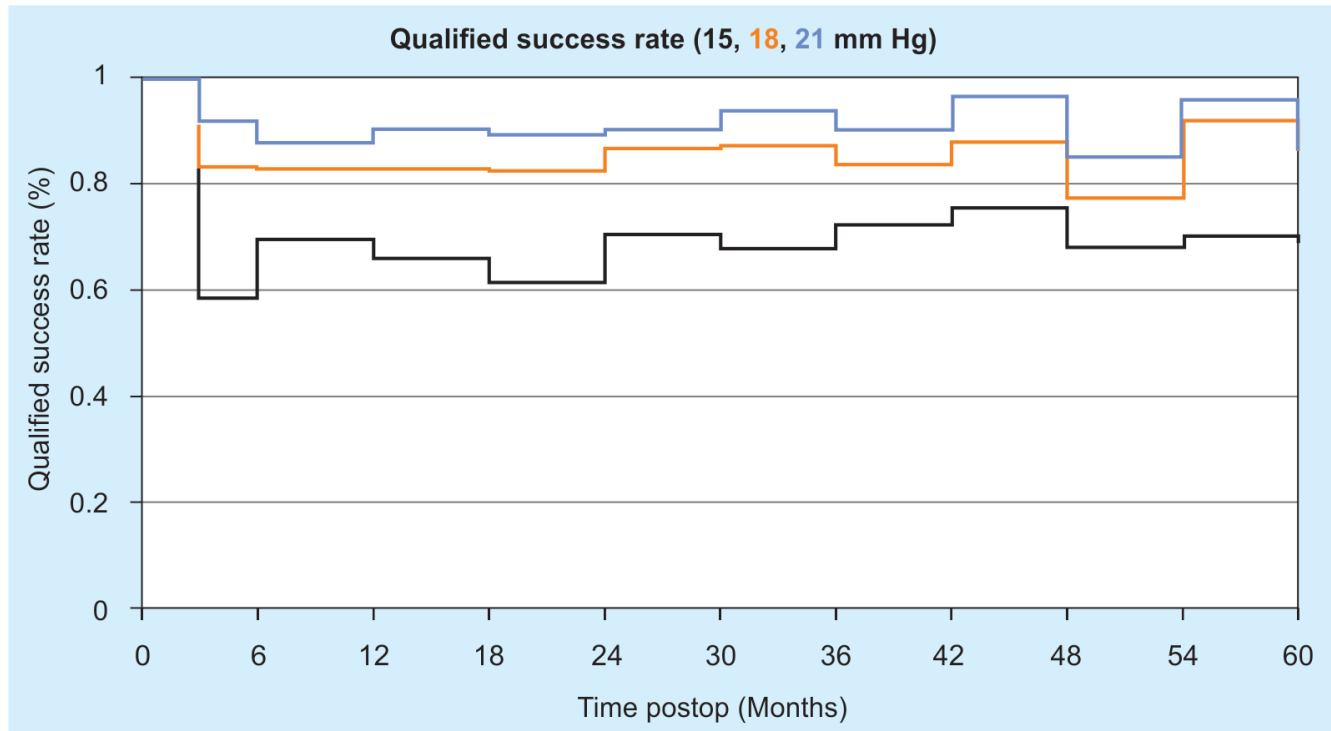

Fig. 3: Long-term qualified success rates with nonpenetrating deep sclerectomy with mitomycin C for three different intraocular pressure cutoff levels (see text for conditions)

Table 2: Comparison of baseline characteristics of eyes that required miostat injections for iris incarceration and eyes that did not

\begin{tabular}{|c|c|c|c|c|c|c|c|c|}
\hline & \multirow[b]{2}{*}{ Age (years) } & \multirow[b]{2}{*}{ Male:female } & \multicolumn{2}{|c|}{ Intraocular pressure (IOP) } & \multicolumn{2}{|c|}{ Medications } & \multirow[b]{2}{*}{$\triangle M D$} & \multirow[b]{2}{*}{$\triangle C P S D$} \\
\hline & & & Preoperative & 5 Years & Preoperative & 5 Years & & \\
\hline Non-miostat eyes & 61.8 & $16: 41$ & 19.8 & 12.4 & 2.76 & 0.54 & -0.33 & 1.13 \\
\hline Miostat eyes & 78.49 & 05:04 & 16.5 & 13.17 & 3.17 & 0.17 & 1.18 & -2.27 \\
\hline$p$ & 0.03 & & 0.33 & 0.72 & 0.38 & 0.2 & 0.96 & 0.24 \\
\hline
\end{tabular}

Additionally, there was no significant difference in preoperative visual field MD and CPSD between these two groups ( $p=0.52$ and 0.52 , respectively).

Humphrey visual field test data which reached reliability criteria at all available pre- and postoperative tests in average MD were compared to average preoperative MD (with standard error of the mean) at each time interval (Fig. 4). The preoperative mean MD was $-12.3 \pm 1.3 \mathrm{~dB}$. At 3 months, MD was $-12.4 \pm 1.7$ $\mathrm{dB},-11.3 \pm 1.6$ at 6 months, $-10.0 \pm 1.5$ at 12 months, $-10.8 \pm$ 1.4 at 18 months, $-12.7 \pm 1.7$ at 2 years, $-10.2 \pm 1.7$ at 2.5 years,
$-14.6 \pm 2.3$ at 3 years, $-11.3 \pm 2$ at 3.5 years, $-10.3 \pm 1.5$ at 4 years, $-11.9 \pm 2.4$ at 4.5 years, and $-12.6 \pm 2$ at 5 years. Thus, the mean MD was better than the preoperative value at $>2 / 3$ of the intervals tested. When MDs at each postoperative interval were compared to preoperative levels, MD values showed no significant deterioration from baseline at any stage, and showed statistically significant improvement at 2.5 years. Intraindividual correlation was strong throughout the study period, suggesting test performance was longitudinally consistent for most patients. $R$ values at all time intervals ranged from 0.83 to 0.94 . 


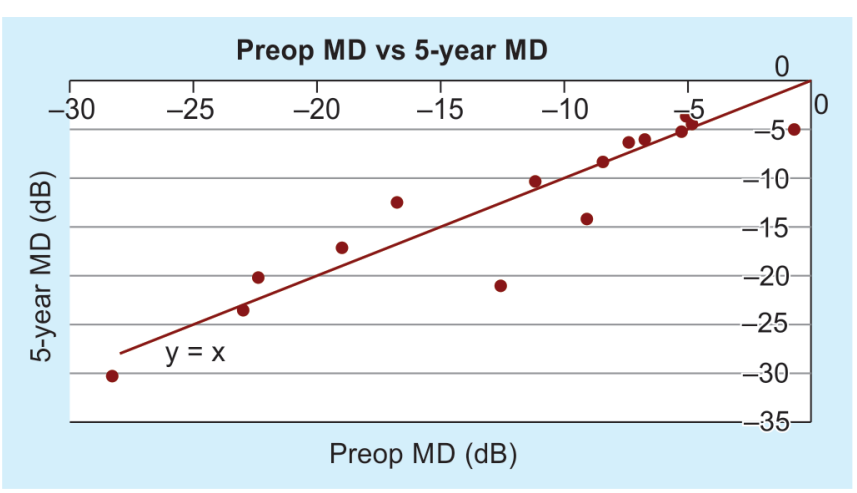

Fig. 4: Comparison of preoperative and postoperative mean deviation (MD); significant difference was not observed $(p>0.1) . R=0.89$ suggests high intra-individual consistency and overall visual field stability. Points above the line $(y=x)$ indicate improved

Corrected pattern standard deviation showed no significant difference at all points except at 2 years and 3.5 years. At both time points, it was increased by $1.4 \mathrm{~dB}$. Intraindividual correlation was again strong, with $R$ values between pre- and postoperative results at all time intervals ranging from 0.83 to 0.92 , indicating strong intraindividual performance consistency across the entire testing period.

The internal analysis was completed to evaluate for attrition bias. Of the 96 eyes with baseline medication and IOP measurements, 34 did not have data at 5 years. Attritional patients had a probability of having IOP greater than 18 that was not significantly different than nonattritional patients $(p>0.25)$, including the 13 eyes that underwent tube shunt procedures. Attrition was also seen for the 42 eyes with baseline visual field data. At some stage throughout the study, 27 of these lacked at least one time-interval dataset. Attrition occurred for a variety of reasons, including insurance was temporarily out of network, monitoring by distant referring eye care clinic lacking HVF device, residential relocation, death, or visual field performance falling outside reliability parameters.

\section{Discussion}

This study found that after NPDS both IOP and antiglaucoma medication use were substantially reduced from preoperative baseline at all postoperative intervals, with high statistical significance (all intervals yielded $p<10^{-5}$ for IOP, and all $p<10^{-14}$ for medications). These tonometric and drug therapeutic advantages were accompanied by remarkable stability in perimetric MD and CPSD on the Humphrey 30-2 analysis, with very high $R$ values for the association between pre- and postoperative MD, providing added statistical confidence by virtue of highly consistent longitudinal results for individual subjects. Humphrey MD in this longer-term study behaved similarly to MD in our previous 18-month study of a different population, with mean follow-up MDs higher than corresponding preoperative values at 8 of 11 time intervals.

Several studies have examined long-term tonometric control of antimetabolite-augmented NPDS and demonstrate maintained IOP control over long periods of time with reduced medication use. ${ }^{5,19,20}$ Results among these studies show remarkable consistency and are similar to those found in this study. This lends credence to the idea that NPDS-MMC allows for satisfactory IOP control, and demonstrates tonometric success over a long period of time.

There is a paucity of data available on visual field survival after NPDS. ${ }^{21}$ Long-term stability of visual acuity has been demonstrated in a small number of studies, but this may not accurately assess glaucomatous damage due to sparing of central vision. ${ }^{5,22}$ One study of 259 eyes by Lachkar et al. demonstrated no average change in visual field parameters (MD, CPSD, corrected loss variance) at 5 years, with only four eyes with glaucomatous visual field progression. ${ }^{5}$ These results are consistent with those in the visual field arm of the present study; both MD and CPSD were stable at 5 years.

The present study has the advantage of following NPDS recipients over a 5 -year period, with very clear, consistent results throughout the medications/IOP portion. The $p$ values related to the reduction of medications and IOP at 5 years are $<10^{-20}$ and $<10^{-6}$, respectively. These reflect significant decrease in both variables even after considering the Bonferroni adjustment for 44 comparisons. Attrition, however, adds a degree of uncertainty to otherwise very robust results. Internal analysis confirmed that these attritional patients showed no significant difference from nonattritional patients when comparing preoperative data to final data, and comparing each patient's final two data points to each other (both $p>0.6$ ). Despite relatively stable MD and CPSD with very stark reductions in IOP and medication use, it is possible that attrition may have marginally skewed the data, especially in the visual field portion of the study, because the fraction of patients who experienced attrition is higher among that group.

More data were available for IOP and medical glaucoma treatment after NPDS surgery than for the visual field portion of this study, so the results from this portion of the study are more conclusive. Of eyes that received NPDS, over $70 \%$ had appropriate IOP without the need for pressure-reducing medication at 5 years, and another $23 \%$ had appropriately controlled IOP with reduced average medication use. Together, this gives a combined qualified and complete success rate of $93 \%$ and illustrates NPDS' ability to remain an effective means for controlling IOP while reducing average medication use over a period of 5 years.

Results can be compared with other NPDS studies that monitored IOP and antiglaucoma medications under various conditions and time periods (Table 3). ${ }^{15-17,23-27}$ This study emphasized tracking visual field survival in the long-term, an obviously very important clinical variable that is curiously not commonly reported in many other studies examining NPDS or other glaucoma-correcting surgeries. ${ }^{28,29}$

The purpose of this study was to continue our ongoing investigation of previously observed recovery of perimetric light sensitivity up to 18 months following IOP reduction in the glaucomatous eye undergoing NPDS. ${ }^{11}$ Throughout the study period, some patients had intervals during which they were on glaucoma medication, and then in subsequent months reverted back to no medication use. The variable nature of medication requirement and the observation that one-third of the eyes in this study necessitated some form of postoperative maintenance demonstrates the importance of regular long-term follow-up for NPDS patients.

Recent studies have demonstrated the fundamental relevance of quantitative analysis of the HVF analysis in glaucoma, confirming that the central nervous system coordinates axonal apoptosis bilaterally to maximize the remaining binocular visual field. ${ }^{30-32}$ Refined data analyzes of electroretinography and visual evoked potentials has further reaffirmed that in progressive glaucoma dynamic function is also under CNS control to maximize binocular function, optimizing complementary high- and lowcontrast input from both afflicted eyes. ${ }^{22}$ The present study 


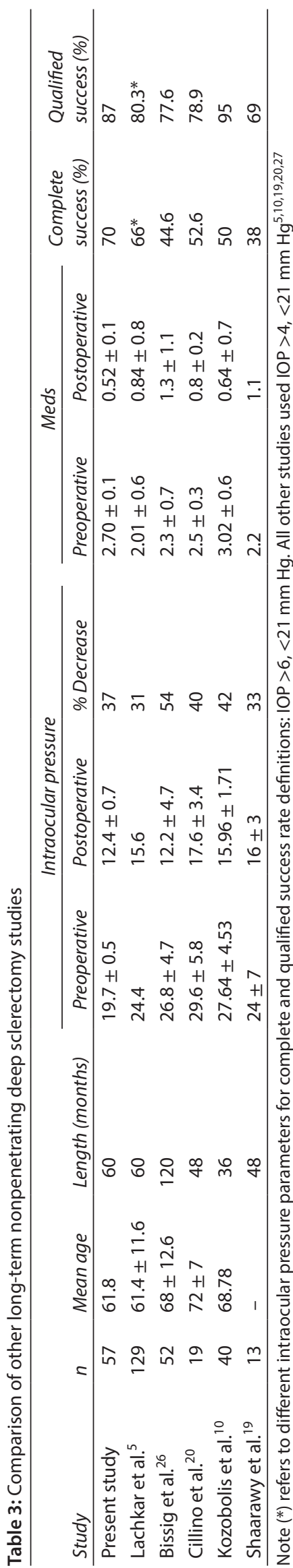

reaffirms that with adequate postoperative management, eyes that have undergone NPDS can maintain adequate IOP control, require low or no medication use, and function with relatively stable visual fields over an extended period of time. Further investigation should compare the visual conservation efficacy of NPDS with other surgical or medical interventions, regardless of their purported sites or mechanisms of action, ${ }^{33}$ to determine the most effective stratagem for preserving bilateral visual function in glaucomatous eyes.

\section{References}

1. Zimmerman TJ, Kooner KS, Ford VJ, et al. Trabeculectomy vs. nonpenetrating deep sclerectomy: a retrospective study of two procedures in phakic patients with glaucoma. Ophthalmic Surg 1984;15(9):734-740.

2. Mermoud A, Schyder CC. Nonpenetrating filtering surgery in glaucoma. Curr Opin Ophthalmol 2000;11(2):151-157. DOI: 10.1097/00055735-200004000-00015.

3. Neudorfer M, Sadetzki S, Anisimova S, et al. Nonpenetrating deep sclerectomy with the use of adjunctive Mitomycin C. Ophthal Surg Lasers Imag Ret 2004;35(1):6-9. DOI: 10.3928/1542-8877-20040101032.

4. Chiselita D. Non-penetrating deep sclerectomy versus trabeculectomy in primary open angle glaucoma surgery. Eye 2001;15(Pt 2):197-201. DOI: 10.1038/eye.2001.60.

5. Lachkar Y, Neverauskiene J, Jeanteur-Lunel MN, et al. Nonpenetrating deep sclerectomy: a 6-year retrospective study. Eur J Ophthalmo 2004;14(1):26-36. DOI: 10.1177/112067210401400105.

6. Roy S, Mermoud A. Deep sclerectomy. Glaucoma Surg 2017;59:36-42. DOI: $10.1159 / 000458484$.

7. Watson PG, Jakeman C, Ozturk M, et al. The complications of trabeculectomy (a 20-year follow-up). Eye 1990;4(3):425-438. DOI: 10.1038/eye.1990.54

8. Bahar I, Kaiserman I, Trope GE, et al. Non-penetrating deep sclerectomy for glaucoma surgery using the femtosecond laser: a laboratory model. Brit J Ophthalmol 2007;91(12):1713-1714. DOI: 10.1136/bjo.2007.116632.

9. Guedes RAP, Guedes VMP, Chaoubah A. Factors associated with non-penetrating deep sclerectomy failure in controlling intraocular pressure. Acta Ophthalmol 2011;89(5):58-61. DOI: 10.1111/j.17553768.2009.01630.

10. Kozobolis VP, Christodoulakis EV, Tzanakis N, et al. Primary deep sclerectomy versus primary deep sclerectomy with the use of mitomycin C in primary open-angle glaucoma. J Glaucoma 2002;11(4):287-293. DOI: 10.1097/00061198-20020800000003.

11. Sponsel WE, Groth SL. Mitomycin-augmented non-penetrating deep sclerectomy: preoperative gonioscopy and postoperative perimetric, tonometric and medication trends. Brit J Ophthalmol 2013;97(3):357-361. DOI: 10.1136/bjophthalmol-2012-301886.

12. Suominen S, Harju M, Kurvinen L, et al. Deep sclerectomy in normaltension glaucoma with and without Mitomycin-c. Acta Ophthalmo 2013;92(7):701-706. DOI: 10.1111/aos.12305

13. Sponsel WE, Harrison J, Elliott WR, et al. Dorzolamide hydrochloride and visual function in normal eyes. Am J Ophthalmol 1997;123(6): 759-766. DOI: 10.1016/s0002-9394(14)71124-9.

14. Armstrong RA. When to use the Bonferroni correction. J Coll Optometr 2014;34(5):502-508. DOI: 10.1111/opo.12131.

15. Arruabarrena C, Munoz-Negrete FJ, Marquez C, et al. Results of nonpenetrating deep sclerectomy in inflammatory glaucoma: one year follow up. Arch Spanish Soc Ophthalmol 2007;82(8):483-488. DOI: 10.4321/s0365-66912007000800006.

16. Iwao $\mathrm{K}$, Inatani $\mathrm{M}$, Seto $\mathrm{T}$, et al. Long-term outcomes and prognostic factors for trabeculectomy with Mitomycin $\mathrm{C}$ in eyes with uveitic glaucoma: a retrospective cohort study. J Glaucoma 2014;23(2):88-94. DOI: 10.1097/IJG.0b013e3182685167. 
17. Shaarawy T, Nguyen C, Schnyder C, et al. Comparative study between deep sclerectomy with and without collagen implant: long term follow up. Brit J Ophthalmol 2004;88(1):95-98. DOI: 10.1136/ bjo.88.1.95.

18. Lewin A, Brondeel R, Benmarhnia T, et al. Attrition bias related to missing outcome data: a longitudinal simulation study. Epidemiology 2018;29(1):87-95. DOI: 10.1097/EDE.0000000000000755.

19. Shaarawy T, Mermoud A. Deep sclerectomy in one eye vs deep sclerectomy with collagen implant in the contralateral eye of the same patient: long-term follow-up. Eye (Lond) 2005;19(3):298-302. DOI: $10.1038 /$ sj.eye.6701469.

20. Cillino S, Di Pace F, Casuccio A, et al. Deep sclerectomy versus trabeculectomy with low-dosage Mitomycin C: four-year follow-up. Ophthalmologica 2008;222(2):81-87. DOI: 10.1159/000112623.

21. Hondur A, Onol M, Hasanreisoglu B. Nonpenetrating glaucoma surgery: meta-analysis of recent results. J Glaucoma 2008;17(2):139146. DOI: 10.1097/IJG.0b013e31814b98f7.

22. Sponsel WE, Johnson SL, Trevino R, et al. Pattern electroretinography and visual evoked potential provide clinical evidence of CNS modulation of high and low contrast VEP latency in glaucoma. Trans Vis Sci Tech 2017;6(6):6. DOI: 10.1167/tvst.6.6.6.

23. Anand N, Kumar A, Gupta A. Primary phakic deep sclerectomy augmented with mitomycin C: long term outcomes. J Glaucoma 2011;94(1):1571-1576. DOI: 10.1097/IJG.0b013e3181ccb926.

24. Yazgan S, Ates H, Guven Yilmaz S, et al. Long-term results of up to 6 years of mitomycin-c augmented non-penetrating deep sclerectomy for pseudoexfoliation glaucoma. Acta Ophthalmol 2016;94(5):n/a. DOI: 10.1111/j.1755-3768.2016.0250.

25. Ollikainen ML, Puustjärvi TJ, Rekonen PK, et al. Mitomycin $\mathrm{C}$-augmented deep sclerectomy in primary open-angle glaucoma and exfoliation glaucoma: a three-year prospective study. Acta Ophthalmol 2011;89(6):548-555. DOI: 10.1111/j.1755-3768.2009. 01772.

26. Goldsmith JA, Ahmed IK, Crandall AS. Nonpenetrating glaucoma surgery. Ophthalmol Clin North Am 2005;18(3):443-460. DOI: 10.1016/j.ohc.2005.05.008, vii. Review.

27. Bissig A, Rivier D, Zaninetti M, et al. Ten years follow-up after deep sclerectomy with collagen implant. J Glaucoma 2008;17(8):680-686. DOI: 10.1097/IJG.0b013e318182ed9e.

28. Figus M, Lazzeri S, Nardi M, et al. Short-term changes in the optic nerve head and visual field after trabeculectomy. Eye 2011;25(8): 1057-1063. DOI: 10.1038/eye.2011.119.

29. Bertrand V, Fieuws S, Stalmans I, et al. Rates of visual field loss before and after trabeculectomy. Acta Ophthalmol 2014;92(2):116-120. DOI: 10.1111/aos.12073.

30. Sponsel WE, Groth SL, Satsangi N, et al. Refined data analysis provides clinical evidence for central nervous system control of chronic glaucomatous neurodegeneration. Transl Vis Sci Technol 2014;6(3):1-13. DOI: 10.1167/tvst.3.3.1.

31. Sponsel WE, Reilly MA, Maddess T. Definitive response to Denniss and Artes: the paired eyes and brain in one person are one unit. Transl Vis Sci Technol 2015;4(2):8. DOI: 10.1167/tvst.4.2.8.

32. Reilly MA, Villareal A, Maddess T, et al. Refined frequency doubling perimetry analysis reaffirms central nervous system control of chronic glaucomatous neurodegeneration. TransI Vis Sci Technol 2015;4(3):7. DOI: 10.1167/tvst.4.3.7.

33. Toris C, Gelfman C, Whitlock A, et al. Making basic science studies in glaucoma more clinically relevant: the need for a consensus. Ocul Pharmacol Ther 2017;33(7):501-518. DOI: 10.1089/jop. 2017.0001. 